\section{Ulzerationen der Haut, Entzündungen der Nasen- und Bronchialschleimhaut, Fieber und belastungsabhängige Dyspnoe}

\author{
A. Voß ${ }^{1}$, M. Leverkus ${ }^{1}$, M. Jenett ${ }^{2}$, R. Gillitzer ${ }^{1}$, \\ H. Hamm', Eva-B. Bröcker ${ }^{1}$ \\ ${ }^{1}$ Universitäts-Hautklinik Würzburg \\ (Direktorin: Prof. Dr. Eva-B. Bröcker) \\ ${ }^{2}$ Institut für Röntgendiagnostik, Medizinische Poliklinik der \\ Universität Würzburg (Direktor: Prof. Dr. D. Hahn)
}

\section{Anamnese}

Ein 71-jähriger Patient berichtet, dass vor etwa 3 Monaten ein kleines Geschwür am linken Nasenflügel aufgetreten sei, das mit der Zeit an Größe zugenommen und sich durch eine lokale Therapie nicht gebessert habe. Ferner bestehe ein blutiger Ausfluss aus der Nase, ein allgemeines Krankheitsgefühl und Appetitlosigkeit. Des Weiteren habe er Atemnot bei Belastung und seit zwei Monaten eine leichte Bronchitis, sowie in letzter Zeit auch immer wieder erhöhte Temperaturen. Aus der Vorgeschichte sind eine Radiojodtherapie wegen Hyperthyreose, eine chronisch-obstruktive Lungenerkrankung, Z.n. Cholezystektomie, Appendektomie und eine Prostataresektion bei Hyperplasie bekannt. Die Familienanamnese ist unauffällig. Von Beruf ist der Patient Bäckermeister gewesen, anschließend war er im öffentlichen Dienst tätig.

\section{Dermatologischer Befund}

Am linken Nasenflügel besteht ein ca. 1,5 × 1,0 cm großes Ulkus mit erythematösem Randsaum (Abb.1), retroaurikulär rechts ein ca. $1 \mathrm{~cm}$ großes Ulkus mit diskret gerötetem Rand.

\section{Routine-Labor}

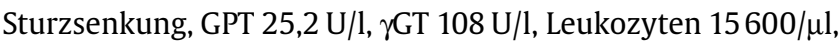
Hb 13,6 mg/dl, Thrombozyten 416000/ $\mu$ l. In der Eiweißelektrophorese Erhöhung der Albuminfraktion auf $182 \mathrm{mg} / \mathrm{d}$ (Norm bis $30 \mathrm{mg} / \mathrm{d}$ ), IgA diskret erhöht auf $434 \mathrm{mg} / \mathrm{dl}$ (Norm bis $400 \mathrm{mg} / \mathrm{dl}$ ),(IgG und IgM unauffällig. Creatinin-Clearance $91,8 \mathrm{ml} / \mathrm{min}$ (Norm 98-156 ml/min), im 24-Stunden-Sammelurin $261 \mathrm{mg} / \mathrm{d}$ Protein (Norm bis $85 \mathrm{mg} / \mathrm{d}$ ). Übrige Routine-Laborparameter unauffällig.

\section{Histologie}

Eine Probebiopsie vom Rand des Ulkus am Nasenflügel zeigt ein gemischtzelliges Entzündungsinfiltrat mit auffällig vielen mehrkernigen Riesenzellen sowohl vom Fremdkörper als auch vom Langhanstyp.

Akt Dermatol 2001; 27: $63-65$

(c) Georg Thieme Verlag Stuttgart $\cdot$ New York ISSN 0340-2541

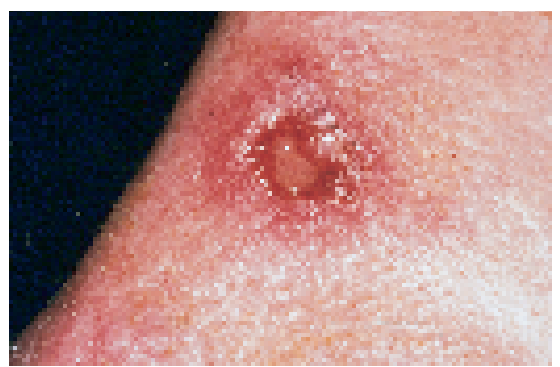

Abb.1 Nasenflügel links: $1,5 \times 1,0 \mathrm{~cm}$ großes Ulkus mit erythematösem Randsaum.

\title{
HNO-Befund
}

Unregelmäßig strukturierte Nasenschleimhaut, V.a. Raumforderung in der Nasennebenhöhle mit Beteiligung der Nasenhaupthöhle (Differentialdiagnose chronische Entzündung versus Tumor).

Rö-Thorax p.a. (Abb. 2)

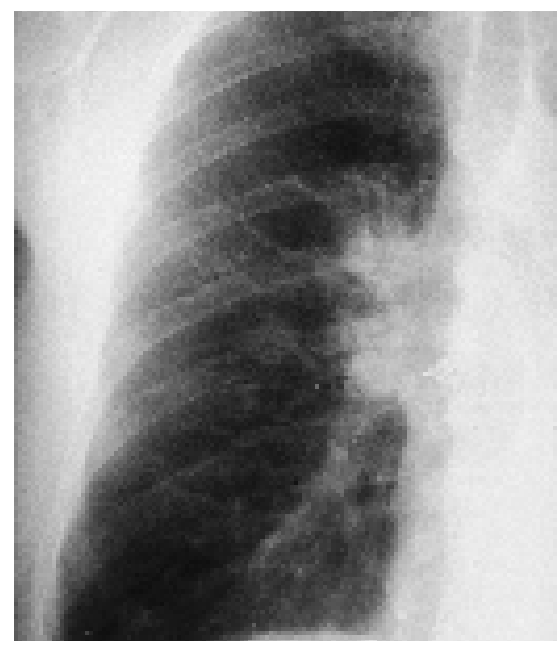

Abb. 2

Rö-Thorax p.a.: Granulom rechts parahilär.

CT-Thorax (Abb. 3)

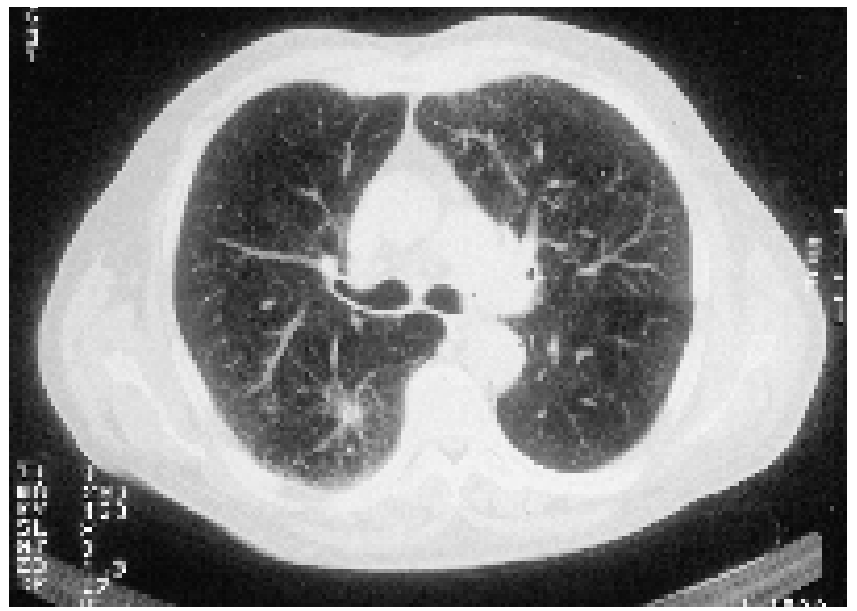

Abb.3 CT-Thorax: Granulom im apikalen Unterlappensegment rechts.

Auflösung auf S. 64 
Auflösung von S. 63

\section{Diagnose: Wegener-Granulomatose}

\section{Weiterführende Labordiagnostik}

Antinukleäre Antikörper negativ, p-ANCA negativ, c-ANCA positiv, Titer $1: 640$ (Normwert: $1:<10$ ), PR3-ANCA positiv Titer $84 \mathrm{IU} / \mathrm{ml}$ (Normwert: < $4 \mathrm{IU} / \mathrm{ml}$ ).

\section{Therapie und Verlauf}

Unsere klinische Verdachtsdiagnose konnte mittels der serologischen Untersuchungen bestätigt werden. Wegen der im Vordergrund stehenden Lungensymptomatik und des zunehmenden Fiebers wurde der Patient in die Medizinische Poliklinik der Universität Würzburg verlegt. Die Therapie wurde mit Cyclophosphamid $150 \mathrm{mg} / \mathrm{tgl}$. und Prednisolon $100 \mathrm{mg} / \mathrm{tgl}$. begonnen. Unter Dosisreduktion auf $100 \mathrm{mg}$ Cyclophosphamid tgl. kam es zu einem Rezidiv, deshalb erfolgte eine erneute Dosissteigerung auf $150 \mathrm{mg}$. Ein halbes Jahr später trat erneut ein Rezidiv in der Nasenhaupthöhle auf, die Cyclophosphamid Dosis wurde daraufhin auf $175 \mathrm{mg}$ erhöht. Zuletzt erfolgte bei stabilem Krankheitsverlauf eine Umstellung auf Methotrexat $15 \mathrm{mg}$ einmal pro Woche i.v.

\section{Kommentar}

Die Wegener-Granulomatose wurde erstmalig von Klinger 1931 beschrieben [1], jedoch nachfolgend von Wegener 1936 als eigenständige Entität abgegrenzt [2,3]. Charakterisiert ist die Erkrankung durch eine nekrotisierende Vaskulitis mittelgroßer und kleiner Arterien und Venen sowie durch destruierende Granulome. Typischer serologischer Befund der Wegener-Granulomatose sind Antikörper gegen zytoplasmatische Antigene von Neutrophilen (c-ANCA, zytoplasmale Anti-Neutrophilen-Zytoplasma-Antikörper).

Es kann sich primär eine Multisystemkrankheit manifestieren oder aber zunächst ein Initialstadium mit Beschränkung auf den Hals-Nasen-Ohren-Raum bestehen [5], das erst nach Monaten oder Jahren in ein generalisiertes Krankheitsbild übergeht, wobei der Befall von Niere und Lunge für die Prognose bestimmend ist. Typische Initialveränderungen sind eitrig-hämorrhagische Rhinitis und Sinusitis oder Otitis media. Später entwickeln sich Granulome mit Destruktionen, die z.B. zur Sattelnase oder Nasenseptumperforation führen. Lungenbeteiligung mit mehr oder weniger produktivem Husten oder restriktiver Lungenfunktionseinschränkung ist ebenfalls häufig. Die Patienten fallen röntgenologisch mit Granulomen, Infiltraten, Kavernenbildung und Pleuraergüssen auf. Die Niere ist typischerweise in Form einer fokal segmentalen Glomerulonephritis betroffen, die in eine generalisierte Glomerulonephritis übergehen kann (Symptome: Hämaturie, Proteinurie, Verlust funktionstüchtiger Nephrone, Niereninsuffizienz und Urämie). Befallen werden auch die Augen mit Konjunktivitis, Episkleritis, Dakryozystitis und Neuritis N. optici, sowie das ZNS mit Mononeuritis multiplex und Vaskulitis der zerebralen Arterien.

30-50\% der Patienten weisen Hautveränderungen auf [7]. Diese können dem generalisierten Erkrankungsprozess vor- ausgehen [6], häufiger jedoch ist eine Hautbeteiligung in fortgeschritteneren Erkrankungsstadien und bei gleichzeitiger Nierenbeteiligung [6]. Die kutanen Manifestationen sind vielfältig, beschrieben sind palpable Purpura, Papeln, hämorrhagische Blasen, Ulzerationen, subkutane Knoten, Pusteln, Bläschen, Makulae, Petechien [7] und Pyoderma gangraenosumähnliche Ulzerationen [6]. Am häufigsten sind die unteren Extremitäten, Gesicht und Brust betroffen.

Histologisch zeigen sich nekrotisierende Vaskulitis und Granulome, wobei sich letztere intramural oder auch außerhalb der Gefäße befinden können. Sie sind histiozytär-epitheloid-leukozytär und oft mit reichlich Riesenzellen durchsetzt.

Die Pathogenese der Erkrankung ist nicht geklärt, ein Autoimmunmechanismus liegt jedoch sehr nahe. Als Auslöser werden neben Umweltfaktoren wie Fremdsubstanzen oder Toxinen auch mikrobielle Trigger diskutiert. So wurde eine Korrelation zwischen der nasalen Besiedelung mit Staphylococcus aureus bei Patienten mit Wegener-Granulomatose und der Erkrankungsaktivität im Hals-Nasen-Ohren-Raum bei diesen Patienten beschrieben [8]. Außerdem scheint auch eine genetische Prädisposition in der Pathogenese der Erkrankung bedeutsam zu sein [8].

Der Wegener-Granulomatose zugrunde liegt eine ANCA-assoziierte nekrotisierende Vaskulitis. Die ANCA sind eine Gruppe von Autoantikörpern vorwiegend der Subklassen IgG1 und $\operatorname{IgG4}$, die gegen lysosomale Proteine in neutrophilen Granulozyten gerichtet sind. C-ANCA sind hochspezifisch für die Wegener-Granulomatose. Über die Titerhöhe ist eine Überwachung der Krankheitsaktivität möglich. Patienten in aktiven Erkrankungsphasen zeigen sehr viel häufiger positive und höhere Titer als Patienten in Remissionsphasen [6]. Die Bestimmung erfolgt mittels indirekter Immunfluoreszenztechnik. Anhand des Fluoreszenzmusters, das durch die unterschiedliche Lokalisation der Zielantigene bestimmt wird, können cANCA (zytoplasmatische Färbung) von p-ANCA (vorwiegend perinukleäre Färbung) unterschieden werden. Die Bedeutung einer dritten Gruppe (weder zytoplasmatisches noch perinukleäres Muster) ist noch nicht geklärt. Weiterhin können ANCA auch durch ELISA-Untersuchungen bestimmt werden, bei denen die Zielantigene z.B. Proteinase 3 oder Myeloperoxidase nachgewiesen werden können. ANCA finden sich außer bei der Wegener-Granulomatose, für die die c-ANCA spezifisch sind, bei primären Systemvaskulitiden, Kollagenosen und anderen System- oder Infektionskrankheiten (hier häufiger pANCA).

Bezüglich der Entstehung der ANCA-assoziierten Vaskulitis wird diskutiert, dass aktivierte Leukozyten primär intrazellulär gelegene Antigene der ANCA auch auf ihrer Zelloberfläche exprimieren. Zirkulierende ANCA binden dann sowohl an ihr Zielantigen als auch an die Fc-Rezeptoren der Leukozyten und es kommt zu deren Aktivierung mit Ausschüttung der lysosomalen Produkte. Die Wirkung wird durch eine Adhäsion der Neutrophilen an die Endothelzellen verstärkt [9]. Experimentell belegt werden konnte der direkte pathogenetische Einfluss der ANCA bislang jedoch nicht sicher [8].

Andere Laborbefunde sind abhängig vom Phasenverlauf der Erkrankung, so z.B. die Erhöhung der Entzündungsparameter wie BSG und Akutphasenproteine, Leuko- und Thrombozytose 
oder hypochrome Anämie. Außerdem können pathologische Laborwerterhöhungen den befallenen Organen entsprechend bestehen.

Therapeutisch ist eine immunsuppressive Medikation notwendig. Üblich ist die gleichzeitige Gabe von Cyclophosphamid $(2 \mathrm{mg} / \mathrm{kg} / \mathrm{d})$ und Prednisolon $(1 \mathrm{mg} / \mathrm{kg} / \mathrm{d})$. Bei klinischem Ansprechen wird die Prednisolon-Dosis langsam reduziert; die Cyclophosphamidtherapie wird meistens für ein halbes bis ein Jahr fortgeführt und dann sehr langsam reduziert. Eine Alternative stellt die i.v. Pulstherapie mit Cyclophosphamid dar (z.B. $500 \mathrm{mg} / \mathrm{m}^{2} \mathrm{KO}$ alle 4 Wochen, bei Nichtansprechen Erhöhung auf $1000 \mathrm{mg} / \mathrm{m}^{2}$ möglich). Zur Einsparung von Kortikosteroiden werden außerdem Methotrexat und Azathioprin verwendet. Durch diese therapeutischen Optionen hat sich die Prognose der früher lebensbedrohlichen Erkrankung heute wesentlich verbessert.

\section{Literatur}

${ }^{1}$ Klinger H. Grenzformen der Periarteriitis nodosa. Frankf Z Pathol 1931; 42: 455-480

${ }^{2}$ Wegener F. Über generalisierte, septische Gefäßerkrankungen. Verh Dtsch Path Ges 1936; 29: $202-210$

${ }^{3}$ Wegener F. Über eine eigenartige rhinogene Granulomatose mit besonderer Beteiligung des Arteriensystems und der Nieren. Beitr Pathol Anat 1939; 102: 37-68

${ }^{4}$ Davies DJ, Moran JE, Niall JF, Ryan GB. Segmental necrotising glomerulonephritis with antineutrophil antibody: possible arbovirus aetiology? Br Med J (Clin Res Ed) 1982; 285: 606-610

${ }^{5}$ Carrington CB, Liebow AA. Limited forms of angiitis and granulomatosis of Wegener's type. Am J Med 1966; 41: 497-527

${ }^{6}$ Daoud MS, Gibson LE, De Remee RA, Specks U, el-Azhary RA, Su WP. Cutaneous Wegener's granulomatosis: clinical, histopathologic, and immunopathologic features of thirty patients. J Am Acad Dermatol 1994; 31: 605-612

${ }^{7}$ Patten SF, Tomecki KJ. Wegener's granulomatosis: cutaneous and oral mucosal disease. J Am Acad Dermatol 1993; 28: 710-718

${ }^{8}$ Hewins P, Tervaert JW, Savage CO, Kallenberg CG. Is Wegener's granulomatosis an autoimmune disease? Curr Opin Rheumatol 2000; $12: 3-10$

${ }^{9}$ Csernok E, Müller A, Gross WL. Immunopathology of ANCA-associated vasculitis. Intern Med 1999; 38: 759-765

\section{Dr. Ariane Voß}

Klinik für Haut- und Geschlechtskrankheiten Universität Würzburg

Josef-Schneider-Str. 2

97080 Würzburg

E-mail: Voß-a.derma@mail.uni-wuerzburg.de 